\title{
Estudio de Tricología Forense en casos donde el Elemento Piloso es Crucial para la Investigación, como Indicio en un Hecho Delictivo
}

\author{
Forensic Trichology Study in cases where the Pilose Element is Crucial to the \\ Investigation, as a Sign in a Criminal Fact
}

Mariuxi Elizabeth Peña Morejón. ${ }^{1}$, Marcia Alexandra Cepeda Paca. ${ }^{2}$, Verónica Paulina Cáceres Manzano. ${ }^{3}$ \& Freddy Rodolfo Sànchez Polit. ${ }^{4}$

Recibido: 10-02-2019 / Revisado: 15-02-209 /Aceptado: 04-03-2019/ Publicado: 12-03-2019

\begin{abstract}
.
DOI: https://doi.org/10.33262/cienciadigital.v3i1.1.404

Within the Forensic Area, the study or analysis of the hair element contributes favorably to the investigation of a Criminal Offense, which is presented as a complementary test, which will later be presented as evidence before a trial, through expert reports. The present work is a bibliographical investigation in which it approaches "Study of Forensic Trichology in cases where the Pilose Element is Crucial for the Investigation, like Indicia in a Delictive Event". This investigation is carried out to identify the importance of the study of a hair element and thus allow to demonstrate a crime mainly violent crimes, cleaning and elimination of foreign particles. Issues related to criminal issues are reviewed and the most important methods and / or techniques that can be performed on this type of evidence were investigated.
\end{abstract}

Keywords: Study, Trichology, Pilose Element, Criminal Acts.

\section{Resumen.}

Dentro del Área Forense el estudio o análisis del elemento piloso contribuye de manera favorable a la investigación de un Hecho Delictivo plasmándose como una prueba complementaria, que posteriormente se presentara como evidencia ante un juicio, por medio de informes periciales.

\footnotetext{
${ }^{1}$ Cruz roja del Tema, Laboratorio clínico, Napo, Ecuador, mariuxielizabeth28@gmail.com

${ }^{2}$ Cruz roja del Tema, Laboratorio clínico, Napo, Ecuador, alexandracepeda12@gmail.

${ }^{3}$ Centro Florence Ambato, Ambato, Ecuador, verito17_caceres85@hotmail.

${ }^{4}$ Policía Nacional, Riobamba. Chimborazo, Ecuador freddy_sanchez@ hotmail.es
} 
El presente trabajo es una investigación bibliográfica en el cual aborda "Estudio de Tricología Forense en casos donde el Elemento Piloso es Crucial para la Investigación, como Indicio en un Hecho Delictivo". Esta investigación se realiza para identificar la importancia del estudio de un elemento piloso y así permitir demostrar un delito principalmente crímenes violentos, la limpieza y eliminación de partículas extrañas. Se revisan temas referentes a los aspectos criminalísticos y se investigaron los métodos y/o técnicas más importantes que se le pueden realizar a este tipo de indicio.

Palabras claves: Estudio, Tricología, Elemento Piloso, Hechos Delictivos.

\section{INTRODUCCIÓN}

Nuestro cabello, lo lavamos, cortamos, teñimos, ondulamos, alisamos o lo sometemos a cualquier cambio físico o cosmético posible. Pero, ¿Nos hemos preguntado cómo se conforma nuestro cabello? ¿Servirá para algo más que cubrirnos y darnos calor? Mirando un poco más allá ¿Servirá para esclarecer un crimen o para ayudar como evidencia en la búsqueda de la verdad?

En efecto, el cabello es de mucha utilidad hoy en día para la ciencia forense sobre todo por su fácil obtención y porque es una matriz segura a la hora de su manipulación.

Son numerosos los ejemplos de casos criminales que han sido resueltos gracias a los elementos pilosos encontrados en la escena del crimen, por lo que la ciencia forense actual se ha volcado al estudio de la tricología forense.

Con la comprensión de que el abordaje forense es un eslabón en la cadena de atención de las víctimas, trabaja en forma integrada con las instituciones de los sectores de justicia, en la construcción de procesos integrados e integrales de atención a las víctimas, con los aportes mutuos desde el conocimiento y la experiencia de todos.

El presente Proyecto de investigación que tiene en sus manos es la cristalización del trabajo y de la entrega personal, contribuyendo así con los estudiantes en esta disciplina.

\section{“Los indicios son testigos mudos que no mienten, sólo hay que hacerlos hablar".} Edmond Locard".

\section{Materiales y Métodos}

Nuestra investigación es de tipo Descriptiva y Explicativa, con el que se pudo establecer conclusiones por método inductivo, a su vez esta es retrospectiva y transversal porque al realizar el presente estudio se recolectaron los datos de los registros del C.I.C.F. Tungurahua; toda la información en la investigación se obtuvo mediante el análisis bibliográfico extraído de una fuente secundaria y datos generados por C.I.C.F. Tungurahua. 


\section{Resultados y Discusión}

Se utilizó la observación y recolección de datos estadísticos de los casos reportados en el Centro de Investigación de Ciencias Forenses-Tungurahua como se observa en la Tabla 1 el número de casos en los que se estudió el elemento piloso es un total de 45 casos, que representan el $100 \%$. Se obtuvieron los siguientes datos: Enero, 5 casos (11\%); Febrero, 9 casos (20\%); Marzo, 1 caso (2\%); Abril, 0 caso (0\%); Mayo, 5 casos (11\%);Junio, 3 casos (7\%); Julio, 5 casos (11\%); Agosto, 2 casos (5\%); Septiembre, 1 caso (2\%); Octubre, 3 casos (7\%); Noviembre, 5 casos (11\%); y Diciembre, 6 casos (13\%).

El elemento piloso es considerado un indicio importante en una gran variedad de crímenes. Las pericias sobre elementos pilosos tienen gran importancia, a pesar de que los investigadores aún no han tomado total conciencia de ello. La búsqueda también es importante. El elemento piloso puede encontrarse:

- En la mano de la víctima.

- En el agresor y particularmente en sus uñas.

- En los órganos de la víctima o del agresor.

- En el lugar de un asesinato (cabellos arrancados) o en el de un robo (pelos caídos o adheridos a un objeto cualquiera).

Detectada la ubicación de los pelos éstos se deben levantar. Habrán de remitirse por separado, en distintos recipientes, los elementos pilosos recogidos en sitios diferentes y, a su vez, los envases irán rotulados con las indicaciones sobre el lugar del hallazgo.

Antes de llevar a cabo este procedimiento, se deben fijar fotográficamente, así como hacer su descripción escrita, complementada con un croquis. En el caso de que se tomen muestras de control, lo que es muy frecuente, deberán obtenerse de varias regiones anatómicas y embalarse por separado.(SANJUAN, 2013)

Los pelos, a pesar de permanecer largos períodos de entierro, estancia en el agua o en medios desfavorables como el jugo gástrico y en ambientes poco húmedos, conservan sus características, lo que permite realizar análisis y comparaciones que orienten en el esclarecimiento de un hecho criminal. (FERRETO, 2005)

La correcta manipulación dependerá de la manera en que se encuentren; sin embargo, se recomienda que cuando se encuentra un indicio de esta naturaleza no se deberá tomar entre los dedos, sino mediante pinzas de goma, teniendo cuidado de no contaminar o alterar de alguna manera las muestras obtenidas y siguiendo una correcta cadena de custodia.

El cabello se debe colocar en pequeñas bolsas de polietileno y sus extremos deberán ser marcados con la letra "a" para la raíz y la letra "b" para la punta (el uso de una lente ayuda en muchos casos a determinar la dirección de la punta y de la raíz del cabello), también pueden utilizarse cajas tipo petri de vidrio, o bien puede utilizarse una cinta con una lado adhesivo para recoger las muestras; posteriormente se desprenden de la cinta con xileno.

En el caso de violaciones deben ponerse mayor énfasis en el pelo púbico. También se 
recomienda recoger las sabanas completas o la ropa de la víctima para después aspirarla.

Para conseguir la muestra de comparación del pelo cuestionado se debe obtener una muestra representativa de cada región del pelo, si no se cuenta con una comparación no se puede realizar el examen ya que será muy difícil decir de quien se trata el pelo y solo se podrá concluir que es un pelo humano. Para el caso del pelo de la cabeza de ser posible, se recomienda tomar alrededor de 10 pelos. (SANJUAN, 2013)

Existen pruebas de naturaleza que nos permiten determinar si es veraderamente es un pelo y se establece por:

a) Técnicas Físicas: Se aplican de acuerdo a la cantidad existente de muestras.

- Fibras vegetales, arden rápidamente, sin olor desagradable, quemándose limpiamente en el extremo afectado.

- Fibras animales, se queman con dificultad, emiten olor a cuerno quemado y su extremos aparece como engrosado en bola o en masa, sus hebras se mantiene individuales.

- Fibras artificiales, arden con mediana velocidad, formando en el extremo afectado una masa compacta uniforme entre todas las hebras.

b) Técnicas químicas: Utilizando una solución saturada de ácido sulfúrico en la que se disuelven los filamentos, a los que luego de ser disueltos se les adiciona dos gotas de solución alcohólica de alfa-naftol, donde:

- Fibras vegetales, adquieren una coloración violeta oscuro.

- Fibras animales, no adquieren ésa coloración.

c) Técnicas microscópicas, Conceden un resultado diagnóstico, pues las fibras vegetales carecen de las estructuras descritas para los pelos (cutícula, corteza, médula) (FERRETO, 2005)

\section{Análisis en laboratorio}

\section{TIPOS DE MUESTRA PARA EL ANÁLISIS}

A. Elementos pilosos del lugar de los hechos

B. Elementos pilosos del cadáver

- Cabello

- Vello púbico

C. Peinado púbico

\section{Materiales}

Los materiales a utilizar dependen de la situación, como por ejemplo si se trata de obtención de muestras de una persona, de una prenda, de un vehículo oen uninmueble.

Por lo general consisten en: 
- Cofia

- Mandil

- Gafas de seguridad o lentes

- Mascarilla

- Guantes

- Pinzas con punta de goma o jebe

- Etiquetas

- Geles fríos, hielo o hieleras

- Tubos cónicos de plástico

- Caja petri

- Papel filtro estándar.

- Peine nuevo

- Cinta adhesivas 3M

- Aspiradora

- Líquido de montaje; por ejemplo si utilizamos agua (índice de refracción "n" igual a 1,33 es demasiado grande la diferencia del índice de refracción de pelo y el índice de refracción del medio de montaje. El medio de montaje debe tener un índice de refracción cerca de la cutícula del cabello y la corteza, entre 1,52 y 1,54. Los medios de montaje pueden aceites minerales servicios o sintéticos, así como resinas semisólidas.

- Estereoscopio

- Microscopio de luztrasmitida

- Microscopio de comparación

La diferencia de los modos de recolección radica en la cantidad de muestras tricológicas; máxima cantidad en el caso de una aspiradora y menos en el caso de las pinzas (pues depende de la capacidad del investigador, así como su experiencia).

\section{Toma de muestra}

Después de remover los escombros de los elementos de prueba, es necesario seleccionar los tipos y cantidad de pelos para el examen. A veces, cuando la eliminación de una gran cantidad de residuos (por ejemplo, por la aspiradora), puede ser necesario seleccionar sólo a una muestra representativa. Este proceso incluye la selección de muestras, tales como pelos de los distintos grupos raciales, área del cuerpo, y el color. Otro método consiste en seleccionar los pelos que son similares en apariencia a un grupo objetivo (por ejemplo, los pelos que se conoce de un sospechoso o la víctima, es decir, buscar cabellos ensortijados, lacios, etc). La combinación de un muestreo aleatorio y el objetivo a seguir asegura a una muestra representativa.

Los cabellos y pelos púbicos muestran una mayor cantidad de características microscópicas que otros pelos humanos, por lo tanto, los pelos de la cabeza y del pubis son rutinariamente comparados. Una adecuada selección de las muestras de cabellos conocidos incluye el arrancamiento con pinzas con punta de goma y el peinado de diferentes zonas cefálicas por separado para ser caracterizado grupalmente. El número de cabellos necesarios para representar una muestra adecuada conocida varía con el individuo. La selección de los pelos para ser montado a partir de una muestra conocida de 
cabellos puede ser al azar, pero deben ser representativos.

La colección de cabellos conocidos de un sospechoso podría tener lugar durante muchos meses, incluso años, después del crimen. En estos casos, las características de la muestra de la cabeza del cabello conocida puede parecer muy diferente de los pelos que se recogieron (del sospechoso) cuando ocurrió el crimen. Algunos examinadores del cabello han indicado que un lapso de tiempo de un año es el límite exterior, y las condiciones ambientales o alteraciones cosméticas podrían hacerla más corta. El vello púbico parece que conservan sus características durante un largo periodo de tiempo. (FERRETO, 2005)

\section{a) Elementos pilosos del lugar de los hechos}

Los elementos pilosos: cabello, ceja, bigote, vello púbico y de la axila, si presentan raíz en fase de crecimiento activo son susceptibles de tipificación por ADN genómico.

En caso de no presentar raíz o que ésta no se encuentre en fase de crecimiento activo, puede ser susceptible de análisis por secuenciación del ADN mitocondrial.

\section{Procedimiento:}

1. Levantar cada elemento piloso con pinzas por la parte media.

2. Depositar en una caja petri. Cada elemento piloso debe ser embalado individualmente.

3. Embalar, etiquetar, y enviar al laboratorio.

4. Para cada levantamiento se debe usar una pinza diferente.

\section{b) Elementos pilosos de cadáver} $>$ cabello

\section{Procedimiento:}

1. Arrancar en promedio 10 cabellos con raíz de tres zonas mejor conservadas del cráneo.

2. Colocar por separado dentro de una caja petri la muestra de cada zona.

3. Embalar y enviar al laboratorio.

Para cada levantamiento se debe usar una pinza diferente.

\section{Procedimiento:}

$$
\text { Vello púbico }
$$

1. Arrancar al menos 10 vellos con raíz de la zona púbica y escroto.

2. Colocar dentro de una caja petri separando cada muestra de cada región.

3. Embalar y enviar.

\section{c) Peinado púbico}

\section{Procedimiento:}

1. Colocar papel de color claro cerca de la región púbica.

2. Peinar la región púbica de manera lenta, uniforme y superficial sin ejercer demasiada presión

3. Agitar el peine sobre el papel para dejar caer los elementos pilosos obtenidos.

4. Colectar cada elemento piloso y depositarlo dentro de una caja petri por separado.

5. Tomar pelo de referencia de la persona o víctima de quien se esté llevando a cabo el peinado.

6. Embalar y enviar al laboratorio. 


\section{Consideraciones:}

- Cuando el elemento piloso se encuentre húmedo, dejar secar antes de introducir en la caja petri. En caso de no disponer de tiempo, colocar en un tubo de plástico cónico; o colocar en papel filtro, pañuelos desechables, servilletas o cualquier tipo de papel absorbente en condiciones de refrigeración hasta su llegada al laboratorio.

- Si el vello púbico se encuentra húmedo, que puede ser con sangre o semen dejar secar, colocar en una en una caja petri y enviar a temperatura ambiente al laboratorio. En el caso de no disponer de tiempo, colocar en un tubo cónico de plástico y mantener en condiciones de refrigeración hasta su llegada al laboratorio. De ser posible recuperar cada fluido para su análisis biológico.

NOTA: Los elementos pilosos susceptibles para análisis de ADN deberán encontrarse en la fase de crecimiento Anágena (fase de crecimiento y elevada actividad celular), o Catágena (fase de madurez con moderada actividad celular (DILLON, 2012)

\section{Muestra dubitada}

Los pelos sueltos deben ser recogidos de los objetos sacándolas de forma individual. Los pelos que están incrustados en o que se adhiera a una persona u objeto debe ser cuidadosamente inspeccionados antes de la extracción. En su caso, la ubicación de estos pelos deben ser cuidadosamente documentado. Se debe tener cuidado de no contaminar, aplastar o romper las muestras.

Los pelos restantes pueden ser recogidos de la ropa, ropa de cama, u otras superficies grandes. Tenga en cuentaque el adhesivo a partir del material de adhesión podría interferir con el análisis de los tratamientos posteriores que puedan realizar en los cabellos. Los pelos sepuedenrecogerde soportes mediante peinado ó por aspirado.

Al recuperar la evidencia de la cabeza de una persona o la región púbica, se puede utilizar la técnica de peinado. Siempre use un peine limpio o uno nuevo. Coloque una hoja de papel limpioenlazonaquesepeinaparaatrapar pelos sueltos. Este documento debe ser incluido en el paquete de pruebas con el peine.

\section{Muestra indubitada}

Se debe obtener los pelos conocidos (o muestras indubitadas) de determinadas regiones somáticas y deben ser caracterizadas grupalmente de cada zona cefálica de las personas relevantes para ser cotejadas con los pelos en duda(muestra desconocida).

Se deben obtener los pelos enteros, con raíces, para que el examinador pueda examinar y compararlos con otros pelos. Debido a que la mayoría de los pelos probablemente ya haya llegado al cese delcrecimiento activo, se puede utilizar procedimientos por separación por peinado para obtener los pelos en la fase de telógena.

Diferentes pelos de una misma área corporal de una persona puede exhibir variación en sus características microscópicas. Por lo tanto, es importante obtener un número suficiente de pelos con el fin de representar de manera adecuada el rango de valores de todas las 
características actuales. Si el margen es amplio, se hace necesario para obtener un gran número de pelos.

Una muestra conocida de cabellos de la cabeza del debe consistir en los pelos de las cinco diferentes áreas del cuero cabelludo (frontal, parietal izquierdo, parietal derecho, vértice y occipital). Las muestras conocidas de cabellos deben de ser obtenidos por una combinación de tracción yel peinado.Idealmente, untotal de 50 pelos se debe obtener del cuero cabelludo. Una muestra de pelos púbicos conocidas o una muestra de cualquier otra región somática idealmente debe consistir de 25 pelos obtenidos por arrancamiento y peinado de diferentes regiones. Se puede realizar con menos de la cantidad recomendada de los pelos, pero esto aumenta la probabilidad de falsa exclusión.

\section{Técnica de montaje}

Para el montaje de muestras indubitadas y/o dubitadas, primero se anota su forma natural y luego se procede a cubrirlas con cinta adhesiva $3 \mathrm{M}$ en una lámina portaobjeto según la cantidad. Se sugiere no montar más de 5 pelos por lámina. Si los pelos sondelongitud inferior a $5 \mathrm{~cm}$, se sugiere colocarlos rectos en la lámina, y si son mayores a $5 \mathrm{~cm}$ se sugiere colocarlos en zig-zag hasta que todo el pelo esté dentro de lalámina.

Luego de asegurar las muestras tricológicas, se sugiere presionar la cinta sobre la muestra para reducir la formación de burbujas que pudiesen dificultar la observación de las características microscópicas.

Otra técnica para la observación de las características microscópicas es la sumersión completa de la muestra en aceite de inmersión para evitar los cambios en los índices de refracción como ocurre con la cinta. (FERRETO, 2005)

El análisis microscópico puede llevarse a cabo con el microscopio de comparación. Para hacer mediciones se puede usar un micrómetro en todos los casos. El medio de montaje para el análisis de pelo, puede ser una resina temporal la cual debe mantener fija al pelo para después cotejar con el pelo de la comparación, cuando se pretenda montar en un medio líquido se puede utilizar ortodiclorobenceno.

El analizador de imagen se maneja en forma manual con movimientos de la platina, a través de la computadora se ve la muestra en el monitor congelando la imagen (15-20 congelaciones) permitiendo de esta forma una mejor observación de la estructura y realizando una comparación del pelo.

La conclusión del análisis se expresa en términos de exclusión por ejemplo: "Existen suficientes características individuales y microscópicas para asociar a una persona con el pelo encontrado en la escena, excluyendo a los demás

\section{Limpieza y eliminación de partículas extrañas.}

Para lavar los pelos se utiliza solución jabonosa o carbonato de potasio $10 \%$. Si fuese muy oscuro, puede emplearse una de las siguientes soluciones:

$>$ Agua oxigenada

$>$ Perhidrol caliente

$>$ Ácido acético 
$>$ Solución hipoclorito de sodio

$>$ Solución alcohólica de cloro

$>$ Ácido nítrico.

\section{Exámenes analíticos del pelo.}

El examen en el laboratorio comprende dos fases:

a) El examen macroscópico.

b) El examen microscópico.

El examen macroscópico permite el conocimiento de su aspecto, color, olor forma, dimensiones, enfermedades, datos de interés pericial. Referente al aspecto, puede estar limpio o impregnado de materia orgánica (sangre o esperma) o bien con parásitos u hongos.

Tomando en cuenta su color, depende, de la cantidad de pigmento como de su distribución, las tonalidades cromáticas van del negro al rojo, pasando por el pardo, el castaño y el rubio, sin dejar de tener en cuenta la carencia de pigmento o decoloración congénita del cabello, generalizada (albinismo) o limitada (pielbaldismo), así como la decoloración fisiológica (canicie). Las decoloraciones artificiales y las coloraciones, dado su extraordinaria frecuencia, son de importancia pericial.

Referente al olor, debido al uso habitual de perfumes empleados indistintamente por ambos sexos, puede tener valor identificativo.

Respecto a la forma, el dato macroscópico es de mayor importancia, por los diversos tipos morfológicos del pelo.

De acuerdo a enfermedades, cuando se demuestre el mismo padecimiento en los pelos del sospechoso y en los de comparación, el dato se puede considerar como muy significativo.

El estudio criminalísticos del pelo es un examen especialmente comparativo entre la muestra problema y las muestras testigo, por lo que se requieren los siguientes microscopios: estereoscópico de comparación con una adaptación para cámara fotográfica, de fluorescencia y binocular de baja potencia, contando con accesorios como son: disco micrométrico, micrómetro de platina, objetivos intercambiables, sistemas para fotografiar indicios.

Los análisis realizados a los pelos humanos en el laboratorio forense se llevan a cabo principalmente mediante microscopia. Esta evaluación implica rutinariamente un proceso de dos etapas la identificación de pelos problemas y de la comparación con pelos testigos; cuya finalidad es comprobar si dos o más individuos estuvieron en contacto con un objeto.

Esta evidencia sociable es particularmente útil en crímenes de violencia, tales como homicidio, asalto sexual, asalto agravado, donde el contacto físico pudo haber ocurrido.

Los crímenes tales como robo con allanamiento de morada y robo armado implican típicamente la recuperación de los artículos y de la ropa que pueden contener los pelos útiles para la identificación de sospechosos. 
El valor de la evidencia del pelo se relaciona con la variabilidad de las características del pelo entre los individuos en la población, que puede ser visualizada con el uso de la microscopia óptica de comparación. Hay factores que afectan la confiabilidad de una asociación del pelo incluyendo experiencia, el entrenamiento, la conveniencia de los estándares sabidos del pelo, y la eficiencia del equipo. El microscopio óptico de comparación consiste en dos microscopios ligeros compuestos conectados por un puente óptico que permite la visión simultánea de pelos desconocidos y de pelos conocidos (La gama de la amplificación usada es aproximadamente 40X a 400X). (SANJUAN, 2013)

Tabla Nº 1 Estadísticas Casos de Tricología Forense.

\begin{tabular}{lrr}
\hline \multicolumn{3}{c}{ CASOS DE TRICOLOGIA EN EL CIFC- } \\
\multicolumn{2}{c}{ TUNGURAHUA EN EL AÑO 2018 } \\
\hline MESES & NUMERO & PROCENTAJE \\
ENERO & 5 & $11 \%$ \\
FEBRERO & 9 & $20 \%$ \\
MARZO & 1 & $2 \%$ \\
ABRIL & 0 & $0 \%$ \\
MAYO & 5 & $11 \%$ \\
JUNIO & 3 & $7 \%$ \\
JULIO & 5 & $11 \%$ \\
AGOSTO & 2 & $5 \%$ \\
SEPTIEMBRE & 1 & $2 \%$ \\
OCTUBRE & 3 & $7 \%$ \\
NOVIEMBRE & 5 & $11 \%$ \\
DICIEMBRE & 6 & $13 \%$ \\
TOTAL & $\mathbf{4 5}$ & $\mathbf{1 0 0 \%}$ \\
\hline
\end{tabular}

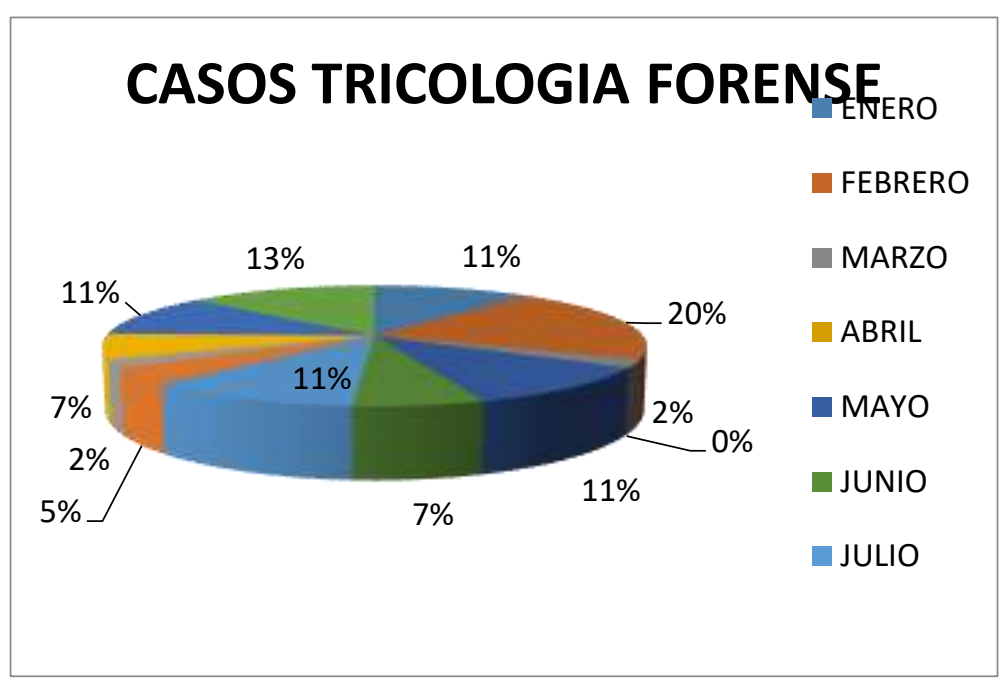

Fuente: https://es.slideshare.net/adnestelamartin/manual-de-tricologia-forense Autores: Mariuxi Peña y Marcia Cepeda 
Anexo $\mathbf{N}^{\mathbf{1}} 1$ Pinzas de goma recta y curva para el arrancamiento de cabellos.

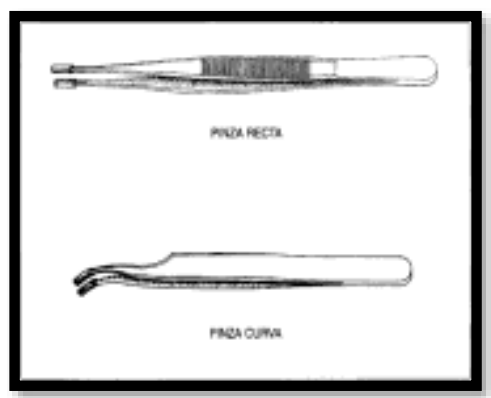

Fuente: https://es.slideshare.net/adnestelamartin/manual-de-tricologia-forense

\section{Autores: Mariuxi Peña y Marcia Cepeda}

Anexo $\mathbf{N}^{\mathbf{0}} 2$ levantamiento de muestras tricológicas mediante pinzas metálicas. Fuente: https://es.slideshare.net/adnestelamartin/manual-de-tricologia-forense

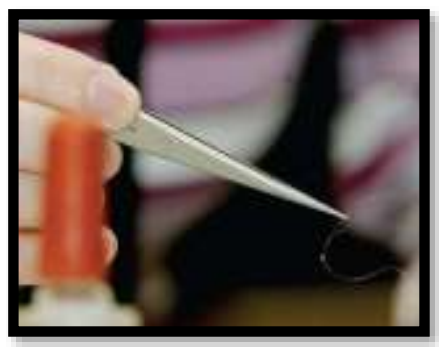

Autores: Mariuxi Peña y Marcia Cepeda

Anexo No 3 forma de extracción de cabellos (izquierda) de las cinco regiones (derecha) para obtener la muestra indubitada

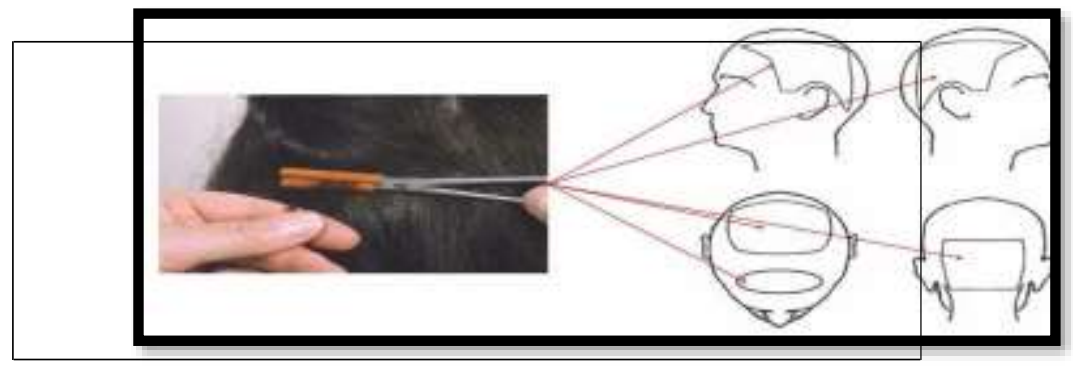

Fuente: https://es.slideshare.net/adnestelamartin/manual-de-tricologia-forense Autores: Mariuxi Peña y Marcia Cepeda 
Anexo $\mathbf{N}^{\circ} 4$ Técnica de Montaje

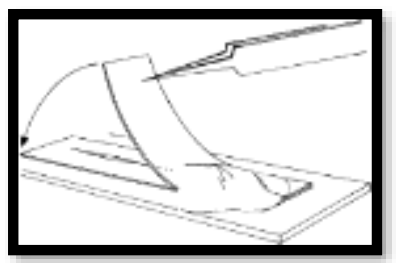

Fuente: https://es.slideshare.net/adnestelamartin/manual-de-tricologia-forense Autores: Mariuxi Peña y Marcia Cepeda

\section{Anexo No 5 Levantamiento del elemento piloso}

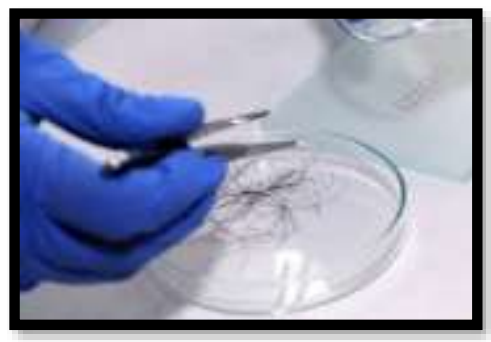

Fuente:https://www.google.com/search?q=ELEMENTO+PILOSO+EN+EL+LUGAR+ DELOS+HECHOS\% 7D\&tbm=isch\&tbs=rimg:CWqNdxTORPcLIjiD0aSBAqg1QIfjH W12STrnjgmLvExAVn0QbyZuwy2COAh6PA7kXhYxXE8iwKp7Owm35aKVscIpioS CYPRpIH4CqDVEfFOeHaYvOshKhIJAhMdbXZJOsRRFdeIPdxCREqEgmeOCYu8T EBWRFuVqj9yQmSoSCfRBvJm7DLYIEa9D3D5levONKhIJ4CHo8DuReFgRAz_1O0 JwR7v8qEgnFcTyLAqns7BGWbMupe8joCioSCSbflopWxwimET6HDnAPYzho\&tbo=

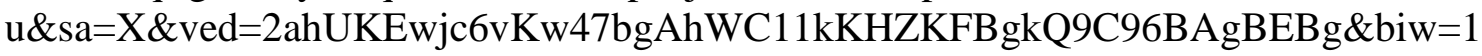
366\&bih=626\&dpr=1\#imgrc=mfWH4LTJhMIvHM:

Autores: Mariuxi Peña y Marcia Cepeda

\section{Anexo $N^{\circ} 6$ Trigrama}

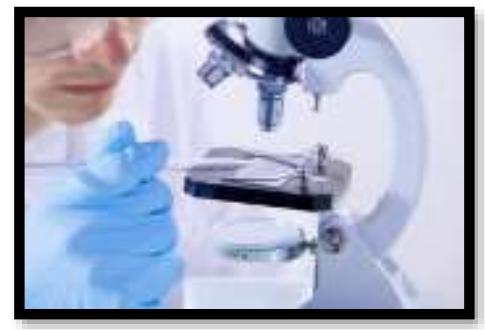

Fuente: https://imdermatologico.com/blogimd/tratamientos-capilares/el-tricograma-labase-del-tratamiento-capilar/

Autores: Mariuxi Peña y Marcia Cepeda 


\section{Anexo $\mathbf{N}^{\circ} 7$ Elementos pilosos en la victima}

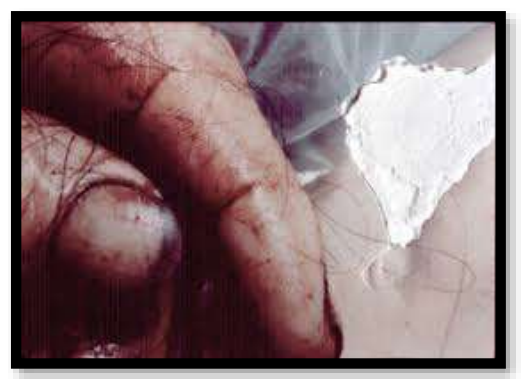

Fuente:https://www.google.com/search?q=ELEMENTO+PILOSO+EN+EL+LUGAR+ DELOS+HECHOS\% 7D\&source=lnms\&tbm=isch\&sa=X\&ved=0ahUKEwiz4fnf37bgA hWntVkKHdihCTcQ_AUIDigB\&biw=1366\&bih=626\#imgrc=ZzJW1_J7FfDXLM:

Autores: Mariuxi Peña y Marcia Cepeda

\section{CONCLUSIONES}

- Mediante el presente trabajo investigativo se logró estudiar la tricología forense en casos donde el elemento piloso es crucial para la investigación, como indicio en un hecho delictivo.

- De acuerdo al estudio de la tricología forense se investigó la metodología que se debe seguir en la recolección de elementos pilosos en un hecho delictivo.

- Por medio de la presente investigación se identificó que análisis se realiza al elemento piloso una vez que ingresa al laboratorio.

\section{AGRADECIMIENTOS}

Agradecemos a las entidades de investigación forense por su colaboración para la realización de la investigación, de igual manera un grato agradecimiento al personal del Área de Biología Forense, por su predisposición a contribuir con nosotros, mediante su experticia profesional compartiendo sus conocimientos.

\section{Referencias Bibliográficas}

\#nocturnovh. (31 de 03 de 2015). ANALISIS NOCTURNO. Recuperado el 03 de 01 de 2019, de ANALISIS NOCTURNO:

http://nocturnovh.wixsite.com/analisisnocturno/singlepost $/ 2015 / 03 / 31 /$ Tricolog\%C3\%ADa-Forense

CAMPANELLI., E. (2014). lalitotowers's Blog. Recuperado el 18 de 12 de 2018, de lalitotowers's Blog: https://lalitotowers.wordpress.com/tricologia-anatomia-tiposde-cabello-cuero-cabelludo-e-infecciones/ 
DILLON, D. M. (2012). PROTOCOLO NACIONAL PARALA TOMA, LEVANTAMIENTO, ENVALAJE Y ENVIO DE INDICIOS Y MUESTRAS BIOLOGICAS A LOS LABORATORIOS FORENSES DE LA REPUBLICA DEL ECUADOR. QUITO: IMPRESION PRODUCCION GRAFICA PRIMERA EDICION .

FERRETO, N. P. (2005). MANUAL DE TRICOLOGIA FORENSE. AREQUIPA: Médica Panamericana.

PADILLA, D. (2019). pelos y fibras criminalistica. Recuperado el 03 de 01 de 2019, de pelos y fibras criminalistica: http://www.academia.edu/15619359/Pelos_y_fibras

SANJUAN, L. M. (MAYO de 2013). PELOS COMO TIPO DE INDICIO EN UN HECHO DELICTIVO. Recuperado el 02 de ENERO de 2019, de PELOS COMO TIPO DE INDICIO EN UN HECHO DELICTIVO: https://www.zaragoza.unam.mx/portal/wpcontent/Portal2015/Licenciaturas/qfb/tesis/tesis_vazquez_sanjuan.pdf

SOTO, V. (01 de 12 de 2013). SOY CRIMINOLOGO. Recuperado el 03 de 01 de 2019, de SOY CRIMINOLOGO: https://criminis.blogcindario.com/2013/12/00167-elpelo.html 


\section{Para citar el artículo indexado.}

Peña M., Cepeda M, Cáceres V.\& Sànchez F. (2019) Estudio de Tricología Forense en casos donde el Elemento Piloso es Crucial para la Investigación, como Indicio en un Hecho Delictivo. Revista electrónica Ciencia Digital 3(1.1), 184-193. Recuperado desde: http://cienciadigital.org/revistacienciadigital2/index.php/CienciaDigital/article/view/404/916

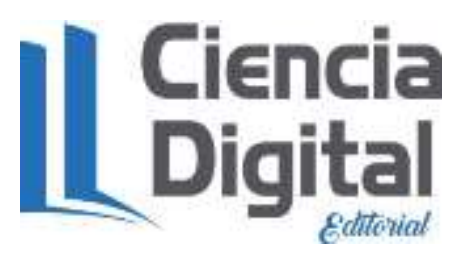

El artículo que se publica es de exclusiva responsabilidad de los autores y no necesariamente reflejan el pensamiento de la Revista Ciencia Digital.

El articulo queda en propiedad de la revista y, por tanto, su publicación parcial y/o total en otro medio tiene que ser autorizado por el director de la Revista Ciencia Digital.
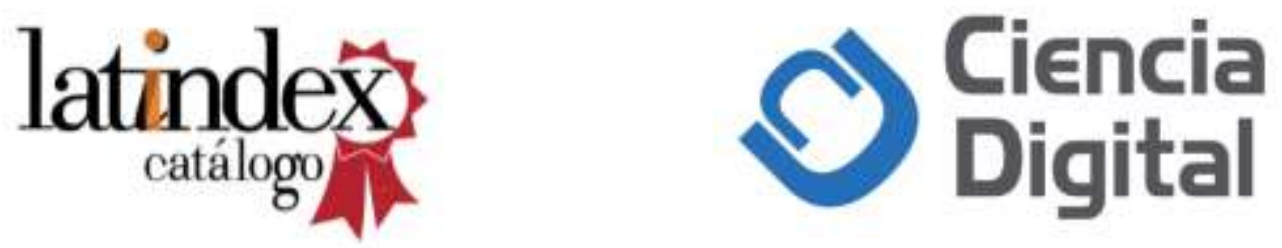